\title{
Convergent Properties of Riccati Equation with Application to Stability Analysis of State Estimation
}

\author{
X. Cai, ${ }^{1}$ Y.S. Ding, ${ }^{1}$ and S. Y. Li $^{2}$ \\ ${ }^{1}$ Engineering Research Center of Digitized Textile \& Apparel Technology, Ministry of Education, College of Information Science and \\ Technology, Donghua University, Shanghai 201620, China \\ ${ }^{2}$ Department of Automation, Shanghai Jiao Tong University and Key Laboratory of System Control and Information Processing, \\ Ministry of Education, Shanghai 200240, China
}

Correspondence should be addressed to Y. S. Ding; ysding@dhu.edu.cn

Received 7 April 2017; Accepted 18 May 2017; Published 12 June 2017

Academic Editor: Weihai Zhang

Copyright (C) 2017 X. Cai et al. This is an open access article distributed under the Creative Commons Attribution License, which permits unrestricted use, distribution, and reproduction in any medium, provided the original work is properly cited.

Since the recursive nature of Kalman filtering always results in a growing size of the optimization problem, state estimation is usually realized by use of finite-memory, receding horizon, sliding window, or "frozen" techniques, which causes difficulties on stability analysis. This paper proposes a novel method on selection of an initial covariance matrix and a horizon for the Kalman filter to make sure that a sequence of the closed-loop Kalman filters are stable as time-invariant filters at subsequent time instant. Convergent properties of Riccati Difference Equation (RDE) are first exploited. Based on these properties, sufficient conditions for stability of a sequence of Kalman filters are obtained. Compared with the existent literature, the convergent properties and the stability conditions are less conservative since they provide analytic results and are applicable to more common cases where the RDEs are not monotonic.

\section{Introduction}

In most industrial control applications, the state variables cannot be directly measured, which necessitates state estimation based on the output measurements during the process of state feedback controller design [1, 2]. Among many different kinds of filters, Kalman filter has been widely used in numerous applications to estimate state for systems with noisy measurements and unmeasured process disturbances including signal processing [3], power electronics [4], and navigation [5]. Essentially it provides an optimal recursive solution for the least square state estimation problem and its theoretical properties including stability and performance have been well addressed in many papers.

In the standard Kalman filter, however, the optimal state estimate at current time is determined recursively from the optimal state estimate and output measurement of the previous time instants. This immediately results in a problem of complexity. Since more measurements become available as time goes on, the estimator has to process more data, which leads to a growing size of the problem. In many applications for embedded implementations where memory limitations have to be considered, alternative methods are proposed by use of finite-memory, receding horizon, or sliding window techniques to overcome this shortage. The basic idea is to maintain a constant length of time window and update the information by discarding the oldest sample as the new measurement comes, which keeps the problem size bounded when more measurements are available. In such case, performance and stability issues have to be restudied; see [6,7].

The Riccati equation, as a fundamental tool in linear optimal control [8] and filtering theory, performs important role during the performance and stability analysis of different variations of the Kalman filter. Starting from 1970s, [9] solves the optimal control problem of linear systems with respect to quadratic performance criteria analytically by use of the algebraic Riccati equation. Reference [10] establishes minimality convergence, uniqueness, and stability concerning the discrete-time matrix Riccati equation and proves the policy space approximation. And [11] addresses the convergence properties of the solution of RDE with a special attention to systems that are not stabilizable in the filtering sense. The 
results are applicable to many filtering or control problems of systems with deterministic disturbances. Motivated by filtering problems in short-time Fourier analysis, $[12,13]$ provide a method to guarantee the exponential asymptotical stability of a sequence of the Kalman filters generated from RDE by selecting appropriate initial covariance matrix through the monotonic properties. The results are later extended to the nonmonotonic case in [14]. And [15] summarizes connections between various Riccati equations and the closed-loop stability of linear quadratic optimal control and estimation. Till recently, the Riccati equation has also been applied into addressing distributed estimation problems [16]. By studying the convergence of the estimation error process, distributed Kalman filter is proposed for potentially unstable and large linear dynamic systems.

The current paper is concerned with convergent properties of RDE and its application to closed-loop stability analysis of Kalman filters as time-invariant filters. Different from most of the previous stability results that rely on the monotonic properties of RDE, a novel convergent property of RDE is first presented for an arbitrary initial positive definite matrix. Further convergent properties of RDE are exploited. Based on these properties, detailed stability analysis is performed. The results provide insights on how to select an initial covariance matrix and a horizon $N$ such that the sequence of Kalman filters generated by RDE is stable as long as the filter has a longer horizon than $N$. The results have potential application in solving the stability of other types of state estimators.

Compared with the existent literature, the main contributions of the paper are as follows: (1) novel convergent properties of RDE are exploited. (2) As an application, sufficient conditions on stability of a sequence of closedloop Kalman filters are provided. (3) The results are less conservative since they are analytic and do not rely on the monotonic properties of solutions of RDE.

This paper is organized as follows. Section 2 provides the preliminaries. General convergent properties of the RDE are discussed in Section 3. Stability analysis of Kalman filters is addressed in Section 4. Section 5 concludes this paper.

\section{Preliminaries}

Consider a linear discrete-time stochastic system described as follows:

$$
\begin{aligned}
\mathbf{x}_{i+1} & =F \mathbf{x}_{i}+G \mathbf{w}_{i}, \\
\mathbf{y}_{i} & =H \mathbf{x}_{i}+\mathbf{v}_{i},
\end{aligned}
$$

where $\mathbf{x} \in \mathbb{R}^{n}$ is the state and $\mathbf{y} \in \mathbb{R}^{p}$ is the measured output. We make the following assumption concerning system (1).

Assumption 1. $\mathbf{w}_{i}$ and $\mathbf{v}_{i}$ are zero mean, white, mutually independent Gaussian noise processes with covariance $E\left\{\left(\begin{array}{c}\mathbf{w}_{i} \\ \mathbf{v}_{i}\end{array}\right)\left(\begin{array}{ll}\mathbf{w}_{j}^{T} & \mathbf{v}_{j}^{T}\end{array}\right)\right\}=\left(\begin{array}{cc}\mathrm{Q} & 0 \\ 0 & R\end{array}\right) \delta_{i j}$, where $Q$ and $R$ are positive definite matrices $(R>0, Q>0)$.

The aim of an estimator is to predict the optimal state $\mathbf{x}_{i}$ on the basis of the measured output variables $\left\{y_{k}\right\}$, $k=0, \ldots, i-1$, and the initial knowledge that $\mathbf{x}_{0}$ is a Gaussian random variable with mean $\mathbf{x}_{0}$ and covariance $P_{0}$.

The standard Kalman predictor is described as

$$
\widehat{\mathbf{x}}_{i+1}=F \widehat{\mathbf{x}}_{i}+K_{i}\left(\mathbf{y}_{i}-H \widehat{\mathbf{x}}_{i}\right),
$$

where Kalman gain is given by

$$
K_{i}=F P_{i} H^{T}\left(H P_{i} H^{T}+R\right)^{-1}
$$

and $P_{i}$ is the solution of the discrete filtering RDE

$$
P_{i+1}=F P_{i} F^{T}-F P_{i} H^{T}\left(H P_{i} H^{T}+R\right)^{-1} H P_{i} F^{T}+Q
$$

solved forwards in time with initial conditions $\mathbf{x}_{0}, P_{0}$. The closed-loop state transition matrix of the Kalman filter is

$$
\bar{F}_{i}=F-F P_{i} H^{T}\left(H P_{i} H+R\right)^{-1} H=F-K_{i} H .
$$

Note that if $P_{0} \geq 0$, then the solution $P_{i}$ of (4) is nonnegative definite for all $i$. This follows easily by rewriting (4) as

$$
P_{i+1}=\left(F-K_{i} H\right) P_{i}\left(F-K_{i} H\right)^{T}+K_{i} R K_{i}^{T}+Q .
$$

The following assumption is made to make sure that the corresponding algebraic Riccati equation (ARE) has the unique nonnegative definite solution.

Assumption 2. $(H, F)$ is detectable, $\left(F, Q^{1 / 2}\right)$ is stabilizable, and $H$ has full rank.

Remark 3. According to [15], if Assumptions 1 and 2 hold for system (1), then the solution of the RDE (4) satisfies

$$
\begin{aligned}
& \lim _{i \rightarrow \infty} P_{i}=P>0, \\
& \lim _{i \rightarrow \infty} K_{i}=K,
\end{aligned}
$$

where $P$ is the positive definite solution of the following algebraic Riccati equation (ARE):

$$
P=F P F^{T}-F P H^{T}\left(H P H^{T}+R\right)^{-1} H P F^{T}+Q,
$$

where

$$
K:=F P H^{T}\left(H P H^{T}+R\right)^{-1} .
$$

This indicates that the sequences $\left\{K_{i}\right\}_{i \in \mathbb{N}_{>0}}$ and $\left\{P_{i}\right\}_{i \in \mathbb{N}_{>0}}$ are bounded.

The following lemma characterizes the monotonic property of RDE, which plays important role in stability analysis of optimal filters.

Lemma 4 (see [13]). For the dynamic system (1) satisfying Assumptions 1 and 2 and $P_{i}$ coming from $R D E$ (4), if $P_{i} \geq P_{i+1}$ $\left(P_{i} \leq P_{i+1}\right)$ holds for any $i \geq 0$, then we have $P_{i+k} \geq P_{i+k+1}$ $\left(P_{i+k} \leq P_{i+k+1}\right)$ for all $k \geq 0$. 
The results above only show the monotonic behavior of the sequence $\left\{P_{i}\right\}$. However, the more common case is that $P_{0}$ might be an arbitrary nonnegative definite matrix, in which case these results may no longer hold.

In the following section, novel convergent properties of $\mathrm{RDE}$ are exploited, which is applicable to an arbitrary positive definite matrix $P_{0}$. The properties are quantitative and analytic compared with the previous results and can include them as special cases.

\section{Convergent Properties of RDE}

More general convergent properties of $\left\{P_{i}\right\}$ will be introduced in this section, some of which have been mentioned in [17]. The following lemma shows that, for any two of the positive definite matrices, upper and lower bounds of the ratio between the two matrices can be analytically expressed in terms of a constant $\gamma>1$ and two nonnegative constants $c_{1}$ and $c_{2}$. The detailed proof is provided in [17]. In the following, we just provide a brief proof in order to keep the completeness and continuity of this paper.

Lemma 5 (see [17]). Let $\gamma>1$ be arbitrary. For any positive definite matrices $\Phi_{1}$ and $\Phi_{2}$, there are two nonnegative constants $c_{1}:=c_{1}\left(\gamma, \Phi_{1}, \Phi_{2}\right)$ and $c_{2}:=c_{2}\left(\gamma, \Phi_{1}, \Phi_{2}\right)$ such that the following inequality holds:

$$
\frac{\gamma-1}{\gamma-1+c_{1}} \Phi_{1} \leq \Phi_{2} \leq \frac{c_{2}+\gamma-1}{\gamma-1} \Phi_{1} .
$$

Proof. Denote $\Phi=\Phi_{1}^{-1 / 2} \Phi_{2} \Phi_{1}^{-1 / 2}$, which is also a positive definite matrix. The following inequality holds:

$$
\lambda_{\min }(\Phi) \Phi_{1} \leq \Phi_{2} \leq \lambda_{\max }(\Phi) \Phi_{1} .
$$

Let

$$
\begin{aligned}
& c_{1}=\max \left\{0,\left(\frac{1}{\lambda_{\min }(\Phi)}-1\right)(\gamma-1)\right\}, \\
& c_{2}=\max \left\{0,(\gamma-1)\left(\lambda_{\max }(\Phi)-1\right)\right\} .
\end{aligned}
$$

A simple calculation shows that

$$
\begin{aligned}
& \frac{\gamma-1}{\gamma-1+c_{1}} \Phi_{1} \leq \lambda_{\text {min }}(\Phi) \Phi_{1} \leq \Phi_{2}, \\
& \frac{\gamma-1}{\gamma-1+c_{2}} \Phi_{1} \geq \lambda_{\max }(\Phi) \Phi_{1} \geq \Phi_{2} .
\end{aligned}
$$

This completes the proof.

According to the RDE (6), it is obvious that $P_{i} \geq Q$ holds for all $i>0$. By use of inequality techniques, the following proposition proves the existence of a constant $\gamma>1$, which will be used to quantify the convergent speed of the sequence $\left\{P_{i}\right\}$.

Proposition 6. For a dynamic system (1) satisfying Assumptions 1 and 2, there exists a constant $\gamma>1$ such that for any fixed $M \in \mathbb{N}_{>0}$ and $P_{i}$ coming from the $R D E$ (4), the following inequality holds for all $i=0, \ldots, M-1$ :

$$
P_{i+1}-\gamma\left(F-K_{i} H\right) P_{i}\left(F-K_{i} H\right)^{T} \geq 0,
$$

where $K_{i}$ is defined in (3).

Proof. For any $k=0, \ldots, M-1$, the RDE equation (6) yields the following inequality:

$$
\begin{aligned}
P_{i+1} & -\left(F-K_{i} H\right) P_{i}\left(F-K_{i} H\right)^{T} \geq Q>0 \\
& \Longrightarrow P_{i+1}-\left(F-K_{i} H\right) P_{i}\left(F-K_{i} H\right)^{T} \\
& \geq P_{i+1}^{1 / 2}\left(P_{i+1}^{-1 / 2} Q P_{i+1}^{-1 / 2}\right) P_{i+1}^{1 / 2} .
\end{aligned}
$$

As mentioned in Remark 3 that $\left\{P_{i}\right\}$ is bounded, there exists a constant $\kappa$ such that $0<Q \leq P_{i} \leq \kappa I$ holds for all $i>0$. Let

$$
\begin{aligned}
& \Gamma:=\frac{1}{\kappa} \cdot Q, \\
& \sigma=\lambda_{\min }(\Gamma) .
\end{aligned}
$$

It can be seen that

$$
P_{k+1}^{-1 / 2} Q P_{k+1}^{-1 / 2} \geq \frac{1}{\kappa} \cdot Q=\Gamma \geq \sigma I_{n \times n},
$$

which leads to

$$
P_{i+1}-\frac{1}{1-\sigma}\left(F-K_{i} H\right) P_{i}\left(F-K_{i} H\right)^{T} \geq 0,
$$

by substituting (17) into inequality (15). The proof is completed by selecting $\gamma:=1 /(1-\sigma)$ and verifying $\gamma>1$.

With the help of Proposition 6, the next proposition provides convergent properties of $\left\{P_{i}\right\}$ in terms of constants $\gamma, c_{1}$, and $c_{2}$. It is assumed that $P_{0}>0$. Similar results can be obtained for the case that $P_{0}=0$.

Proposition 7. Consider system (1) satisfying Assumptions 1 and 2 and $P_{0}$ and $P_{1}$ from RDE (4), and there exist a constant $\gamma>1$ and two nonnegative constants $c_{1}=c_{1}\left(\gamma, P_{0}, P_{1}\right)$ and $c_{2}=c_{2}\left(\gamma, P_{0}, P_{1}\right)$ such that for any given fixed $M \in \mathbb{N}_{>0}$ the following inequality holds:

$$
\underline{\beta}_{i} P_{i} \leq P_{i+1} \leq \bar{\beta}_{i} P_{i}, \quad i=0, \ldots, M-1,
$$

where

$$
\begin{aligned}
& \underline{\beta}_{i}:=\frac{\gamma^{i}(\gamma-1)}{\gamma^{i}(\gamma-1)+c_{1}} ; \\
& \bar{\beta}_{i}:=\frac{\gamma^{i}(\gamma-1)+c_{2}}{\gamma^{i}(\gamma-1)} .
\end{aligned}
$$

Proof. Since the proofs of both sides are similar, we only prove the right side of the inequality. According to Proposition 6, it follows that

$$
Q+K_{i} R K_{i}^{T} \geq(\gamma-1)\left(F-K_{i} H\right) P_{i}\left(F-K_{i} H\right)^{T} .
$$


The rest of this part is proved through induction.

The choice of parameters $\left(\gamma, c_{1}, c_{2}\right)$ shows that inequality (19) holds for $k=0$.

Assume that, for any fixed $M, P_{k+1} \leq \bar{\beta}_{i} P_{i}$ holds for $i=m(m=0,1, \ldots, M-2)$. In the following part, we prove that the inequality also holds when $i=m+1$. By using inequality techniques, RDE can be transformed into the following inequality:

$$
\begin{aligned}
P_{m+1}= & \left(F-K_{m} H\right) P_{m}\left(F-K_{m} H\right)^{T}+K_{m} R K_{m}^{T}+Q \\
\geq & \left(F-K_{m} H\right) P_{m}\left(F-K_{m} H\right)^{T}+K_{m} R K_{m}^{T}+Q \\
& +\frac{\left(\bar{\beta}_{m}-1\right)(\gamma-1)}{\bar{\beta}_{m}+\gamma-1}\left(F-K_{m} H\right) P_{m}\left(F-K_{m} H\right)^{T} \\
& -\frac{\bar{\beta}_{m}-1}{\bar{\beta}_{m}+\gamma-1}\left(K_{m} R K_{m}^{T}+Q\right) \\
= & \frac{\gamma \bar{\beta}_{m}}{\bar{\beta}_{m}+\gamma-1}\left(F-K_{m} H\right) P_{m}\left(F-K_{m} H\right)^{T} \\
& +\frac{\gamma}{\bar{\beta}_{m}+\gamma-1}\left(K_{m} R K_{m}^{T}+Q\right) .
\end{aligned}
$$

Since inequality (19) holds at $k=m$, we have

$$
P_{m+1} \leq \bar{\beta}_{m} P_{m}
$$

Substituting into inequality (22) yields

$$
\begin{aligned}
& P_{m+1} \geq \frac{\gamma}{\bar{\beta}_{m}+\gamma-1}\left[\left(F-K_{m} H\right) P_{m+1}\left(F-K_{m} H\right)^{T}\right. \\
& \left.\quad+\left(Q+K_{m}^{T} R K_{m}\right)\right] .
\end{aligned}
$$

According to the Principle of Optimality, it follows that

$$
\begin{aligned}
& P_{m+2}=\min _{K}\left\{(F-K H) P_{m+1}(F-K H)^{T}\right. \\
& \left.+\left(K R K^{T}+Q\right)\right\} .
\end{aligned}
$$

Thus, we have

$$
P_{m+1} \geq \frac{\gamma}{\bar{\beta}_{m}+\gamma-1} P_{m+2}=\frac{1}{\bar{\beta}_{m+1}} P_{m+2},
$$

which implies the inequality holds when $k=m+1$.

The proof of the left side of inequality (19) is similar and has been omitted here.

Remark 8. Proposition 7 proposes a quantitative convergent property of the RDE (4) which has included monotonicity as a special case. For example,

(1) if $P_{0} \leq P_{1}$ holds, we have $c_{1}=0$ by Lemma 5 . Substituting $c_{1}$ into Proposition 7 immediately yields that $P_{i} \leq P_{i+1}$;
(2) if $P_{0} \geq P_{1}$ holds, we have $c_{2}=0$ by Lemma 5 . Substituting $c_{2}$ into Proposition 7 immediately yields that $P_{i} \geq P_{i+1}$.

Moreover, the results are able to deal with arbitrary positive definite matrices of $P_{0}$, which is more applicable than the previous literature.

In the remaining part of this section, more convergent properties of RDE are exploited. A lemma on comparison of RDE solutions is introduced, which shows that the difference between two sequences of RDE solutions also satisfies a new RDE.

Lemma 9 (see [15]). Consider two RDEs with the same $F, H$, and $R$ matrices but possibly different $Q^{\prime} s, Q^{1}$, and $Q^{2}$, respectively. Denote their solution matrices $P_{i}^{1}$ and $P_{i}^{2}$, respectively. Then, the difference between the two solutions $\widetilde{P}_{i}=$ $P_{i}^{2}-P_{i}^{1}$ satisfies the following $R D E$ :

$$
\begin{aligned}
\widetilde{P}_{i+1}= & \widetilde{F}_{i}^{1} \widetilde{P}_{i} \widetilde{F}_{i}^{1 T}-\widetilde{F}_{i}^{1} \widetilde{P}_{i} H^{T}\left(H P_{i}^{2} H^{T}+R\right)^{-1} H \widetilde{P}_{i} \widetilde{F}_{i}^{1 T} \\
& +\widetilde{Q}
\end{aligned}
$$

or

$$
\begin{aligned}
\widetilde{P}_{i+1}= & \widetilde{F}_{i}^{1} \widetilde{P}_{i} \widetilde{F}_{i}^{1 T}-\widetilde{F}_{i}^{1} \widetilde{P}_{i} H^{T}\left(H \widetilde{P}_{i} H^{T}+\widetilde{R}_{i}\right)^{-1} H \widetilde{P}_{i} \widetilde{F}_{i}^{1 T} \\
& +\widetilde{Q},
\end{aligned}
$$

where

$$
\begin{aligned}
\widetilde{F}_{i}^{1} & =F-F P_{i}^{1} H^{T}\left(H P_{i}^{1} H+R\right)^{-1} H, \\
\widetilde{Q} & =Q_{2}-Q_{1}, \\
\widetilde{R}_{i} & =H P_{i}^{1} H^{T}+R .
\end{aligned}
$$

In order to investigate the further properties, the following assumption is made. Novel convergent properties of the difference between two solutions of RDE are addressed on the basis of Lemma 9.

Assumption 10. $(H, F)$ is detectable, $\left(F, \widetilde{Q}^{1 / 2}\right)$ is stabilizable, and $H$ has full rank.

Proposition 11. Consider two RDEs with the same F, $H$, and $R$ matrices. Denote their solution matrices $P_{i}^{1}$ and $P_{i}^{2}$ and the difference between the two solutions $\widetilde{P}_{i}:=P_{i}^{2}-P_{i}^{1}$. For system (1) satisfying Assumptions 1 and 2, if $Q^{2}>Q^{1}$ and $P_{i}^{2}-P_{i}^{1} \geq 0$ hold, then there exist a constant $\tilde{\gamma}>1$ and two nonnegative constants $\widetilde{c}_{1}=\widetilde{c}_{1}\left(\tilde{\gamma}, \widetilde{P}_{0}, \widetilde{P}_{1}\right)$ and $\widetilde{c}_{2}=\widetilde{c}_{2}\left(\widetilde{\gamma}, \widetilde{P}_{0}, \widetilde{P}_{1}\right)$ such that for any fixed $N \in \mathbb{N}_{>0}$ the following inequality holds for all $i=$ $0, \ldots, N-1$ :

$$
\underline{\zeta}_{i} \widetilde{P}_{i} \leq \widetilde{P}_{i+1} \leq \bar{\zeta}_{i} \widetilde{P}_{i}
$$


where

$$
\begin{gathered}
\underline{\zeta}_{i}:=\frac{\widetilde{\gamma}^{i}(\tilde{\gamma}-1)}{\widetilde{\gamma}^{i}(\tilde{\gamma}-1)+\widetilde{c}_{1}} \\
\bar{\zeta}_{i}:=\frac{\tilde{\gamma}^{i}(\tilde{\gamma}-1)+\widetilde{c}_{2}}{\widetilde{\gamma}^{i}(\tilde{\gamma}-1)} .
\end{gathered}
$$

Proof. According to Lemma 9, $\widetilde{P}_{i+1}$ satisfies the RDE

$$
\begin{aligned}
\widetilde{P}_{i+1}= & \widetilde{F}_{i}^{1} \widetilde{P}_{i} \widetilde{F}_{i}^{1 T}-\widetilde{F}_{i}^{1} \widetilde{P}_{i} H^{T}\left(H \widetilde{P}_{i} H^{T}+\widetilde{R}_{i}\right)^{-1} H \widetilde{P}_{i} \widetilde{F}_{i}^{1 T} \\
& +\widetilde{Q},
\end{aligned}
$$

where

$$
\begin{aligned}
\widetilde{F}_{i}^{1} & =F-F P_{i}^{1} H^{T}\left(H P_{i}^{1} H+R\right)^{-1} H, \\
\widetilde{Q} & =Q_{2}-Q_{1}, \\
\widetilde{R}_{i} & =H P_{i}^{1} H^{T}+R .
\end{aligned}
$$

Since $Q^{2}>Q^{1}$ and $P_{i}^{2}-P_{i}^{1} \geq 0$ hold, we have $\widetilde{Q}>0$ and $\widetilde{P}_{i} \geq 0$. RDE (32) can be rewritten as

$$
\widetilde{P}_{i+1}=\left(\widetilde{F}_{i}^{1}-\widetilde{K}_{i} H\right) \widetilde{P}_{i}\left(\widetilde{F}_{i}^{1}-\widetilde{K}_{i} H\right)^{T}+\widetilde{K}_{i} \widetilde{R}_{i} \widetilde{K}_{i}^{T}+\widetilde{Q},
$$

which implies that $\widetilde{P}_{i+1} \geq \widetilde{Q}>0$. The rest of the proof is similar to Proposition 7 and has been omitted here.

Remark 12. Based on the statement in Lemma 9 that the difference between the two solutions of RDE also satisfies a new type of RDE, further convergent properties of the differences between the two solutions are addressed in Proposition 11. Let us also take some special cases as examples.

(1) If $\widetilde{P}_{0} \leq \widetilde{P}_{1}$ holds, we have $\widetilde{c}_{1}=0$. Substituting $\widetilde{c}_{1}=0$ into Proposition 11 immediately yields that $\widetilde{P}_{i} \leq \widetilde{P}_{i+1}$.

(2) If $\widetilde{P}_{0} \geq \widetilde{P}_{1}$ holds, we have $\widetilde{c}_{2}=0$. Substituting $\widetilde{c}_{2}$ into Proposition 11 immediately yields that $\widetilde{P}_{i} \geq \widetilde{P}_{i+1}$.

Actually, the results above have also included some of the existent results. Select $P_{0}^{2}=P_{0}$ and $P_{0}^{1}=P_{1}$, and then we have $P_{1}^{2}=P_{1}$ and $P_{1}^{1}=P_{2}$. According to statement 1 , if $\widetilde{P}_{0} \leq \widetilde{P}_{1}$ holds, which implies that $P_{2}-2 P_{1}+P_{0} \leq 0$, then it follows that $\widetilde{P}_{i} \leq \widetilde{P}_{i+1}$, yielding $P_{i+2}-2 P_{i+1}+P_{i} \leq 0$. This is consistent with results in $[14,15]$.

In addition, the results are able to deal with arbitrary positive definite matrices of $\widetilde{P}_{0}$, which is more applicable than the previous literature.

As an application, convergent properties of $\mathrm{RDE}$ are utilized to investigate stability of a sequence of Kalman filters in the next section.

\section{Application to Stability Analysis of Kalman Filters}

The following result provides a sufficient condition such that a sequence of Kalman filters is stable for all horizons larger than a certain value.
Theorem 13. Let $N$ be a fixed integer. Consider the dynamic system (1) satisfying Assumptions 1 and 2, $P_{0}>0, \gamma$ from Proposition 6, $P_{1}$ from RDE (4), and $c_{2}$ from Lemma 5, and if for the given $N$, the following inequality holds:

$$
\gamma^{N-1}(\gamma-1)^{2}-c_{2}>0
$$

then the closed-loop matrix $\bar{F}_{i}$ from (5) is Hurwitz for all $i \geq$ $N-1$.

Proof. We first prove that $\bar{F}_{N-1}$ is Hurwitz. yields

If $\gamma^{N-1}(\gamma-1)^{2}>c_{2}$ holds, by using Proposition 7 this

$$
\begin{aligned}
P_{N} & \leq \frac{\gamma^{N-1}(\gamma-1)+c_{2}}{\gamma^{N-1}(\gamma-1)} P_{N-1} \\
& <\frac{\gamma^{N-1}(\gamma-1)+\gamma^{N-1}(\gamma-1)^{2}}{\gamma^{N-1}(\gamma-1)} P_{N-1}=\gamma P_{N-1} .
\end{aligned}
$$

On the other hand, it has been proved in Proposition 6 that

$$
P_{N}-\gamma\left(F-K_{N-1} H\right) P_{N-1}\left(F-K_{N-1} H\right)^{T} \geq 0 .
$$

Combining the above two inequalities yields

$$
\left(F-K_{N-1} H\right) P_{N-1}\left(F-K_{N-1} H\right)^{T}-P_{N-1}<0 .
$$

This indicates that $\bar{F}_{N-1}$ is Hurwitz.

Note that the function $\eta\left(\gamma, c_{2}, N\right):=\gamma^{N-1}(\gamma-1)^{2}-c_{2}$ is monotonically increasing with respect to $N$ given that $\gamma>1$ and $c_{2}$ and $N \geq 1$. Under condition (35), it is easy to verify that $\gamma^{i}(\gamma-1)^{2}-c_{2}>0$ holds for all $i \geq N-1$.

Thus, by a similar proof, $\bar{F}_{i}$ is Hurwitz for all $i \geq N-1$.

Remark 14. Theorem 13 provides a sufficient condition on closed-loop stability of a sequence of Kalman filters. Actually, some existent results can be deemed as a special case for condition (35). For example, if $P_{1} \leq P_{0}$, we have $c_{2}=0$ according to Lemma 5. It can be verified that condition (35) holds for all $i \geq 1$, which implies that all the closed-loop filters are Hurwitz. This is consistent with results in [13] which proves stability of Kalman filters based on monotonicity of $\left\{P_{i}\right\}$.

Remark 15. The function $\eta\left(\gamma, c_{2}, N\right):=\gamma^{N-1}(\gamma-1)^{2}-c_{2}$ is monotonically increasing with respect to $N$ for the given $\gamma>$ 1 and $c_{2}$ and $N \geq 1$. This implies that there always exists a positive integer $N^{*}=N^{*}\left(\gamma, P_{0}, P_{1}\right)$ such that, for any $N \geq$ $N^{*}$, condition (35) holds. From this point of view, stability can be guaranteed for the closed-loop system as long as the horizon is larger than a certain value.

Remark 16. The advantage of the proposed method is that stability conditions can be analytically expressed by use of the parameters such as $\gamma, N$, and $c_{2}$. From the proof of Proposition 6 , it is shown that $\gamma$ is determined by $\sup _{k \in \mathbb{N}}\left\{\lambda_{\text {max }}\left(P_{k}\right)\right\}$ (a constant as long as the system model and $Q, R$ had been fixed) and it stands for the convergent speed of the RDE solutions $\left\{P_{i}\right\}$ to the ARE solution $P_{\infty}$. 
In the next part, a corollary is provided based on Proposition 11 .

Corollary 17. Let $\widetilde{N}$ be a fixed integer and $\widetilde{P}_{0}>0$ and $\tilde{\gamma}>1$ come from Proposition 11. For the dynamic system (1) satisfying Assumptions 1 and 10 and the given $\widetilde{N}$, if the following inequality holds:

$$
\widetilde{\gamma}^{\widetilde{N}-1}(\widetilde{\gamma}-1)^{2}-\widetilde{c}_{2}>0
$$

then the closed-loop matrix $\widetilde{F}_{i}^{c}$ is Hurwitz for all $i \geq \widetilde{N}-1$.

Proof. The proof is similar to that of Theorem 13. If $\widetilde{\gamma}^{\widetilde{N}-1}(\widetilde{\gamma}-$ $1)^{2}>\widetilde{c}_{2}$ holds, applying Proposition 11 leads to

$$
\begin{aligned}
\widetilde{P}_{N} & \leq \frac{\widetilde{\gamma}^{\widetilde{N}-1}(\tilde{\gamma}-1)+\widetilde{c}_{2}}{\widetilde{\gamma}^{N-1}(\widetilde{\gamma}-1)} \widetilde{P}_{N-1} \\
& <\frac{\widetilde{\gamma}^{\widetilde{N}-1}(\widetilde{\gamma}-1)+\widetilde{\gamma}^{N-1}(\widetilde{\gamma}-1)^{2}}{\widetilde{\gamma}^{\widetilde{N}-1}(\widetilde{\gamma}-1)} \widetilde{P}_{\widetilde{N}-1}=\widetilde{\gamma}_{\widetilde{N}-1} .
\end{aligned}
$$

On the other hand, the following inequality holds:

$$
\begin{aligned}
\widetilde{P}_{\widetilde{N}} & -\widetilde{\gamma}\left(\widetilde{F}_{\widetilde{N}-1}^{1}-\widetilde{K}_{\widetilde{N}-1} H\right) \widetilde{P}_{\widetilde{N}-1}\left(\widetilde{F}_{\widetilde{N}-1}^{1}-\widetilde{K}_{\widetilde{N}-1} H\right)^{T} \\
& \geq 0
\end{aligned}
$$

Combining two inequalities yields

$$
\begin{aligned}
& \left(\widetilde{F}_{\widetilde{N}-1}^{1}-\widetilde{K}_{\widetilde{N}-1} H\right) \widetilde{P}_{\widetilde{N}-1}\left(\widetilde{F}_{\widetilde{N}-1}^{1}-\widetilde{K}_{\widetilde{N}-1} H\right)^{T}-\widetilde{P}_{N-1} \\
& \quad<0
\end{aligned}
$$

This indicates that $\widetilde{F}_{\widetilde{N}-1}^{c}$ is Hurwitz.

Note that $\tilde{\eta}\left(\widetilde{\gamma}, \widetilde{c}_{2}, \widetilde{N}\right):=\widetilde{\gamma}^{\widetilde{N}-1}(\widetilde{\gamma}-1)^{2}-\widetilde{c}_{2}$ is monotonically increasing with respect to $\widetilde{N}$ for any $\widetilde{\gamma}>1$ and $\widetilde{c}_{2}$ and $\widetilde{N} \geq 1$. Under condition (39), it is easy to verify that $\tilde{\gamma}^{i}(\widetilde{\gamma}-1)^{2}-\widetilde{c}_{2}>0$ holds for all $i \geq \widetilde{N}-1$. 1.

Thus, by a similar proof, $\widetilde{F}_{i}^{c}$ is Hurwitz for all $i \geq \widetilde{N}-$

Remark 18. There exist essential differences between Theorem 13 and Corollary 17 although the proof seems similar, since, by appropriate selection of different sequence of $P_{i}^{2}$ and $P_{i}^{1}$, more details can be obtained.

Also, Corollary 17 can be a supplement to Theorem 13 during the proof on stability of a sequence of Kalman filters. For example, select $P_{0}^{1}=P_{0}$ and $P_{0}^{2}=P_{1}$ and then $P_{1}^{1}=P_{1}$ and $P_{1}^{2}=P_{2}$. If $\widetilde{P}_{0} \geq \widetilde{P}_{1}$ holds, we have $\widetilde{c}_{2}=0$. Combined with other conditions, it can be proved that the closed-loop matrix is stable, which is consistent with Theorem 3.1 in [14].

\section{Conclusions and Future Work}

By exploiting convergent properties of RDE in optimal filtering problems, this paper presents novel convergent properties and applies them to study stability analysis of a sequence of Kalman filters. These results have fewer requirements on the initial covariance matrix and thus are applicable to a wider case. Future work includes applying the convergent properties to stability analysis of other kinds of estimators.

\section{Conflicts of Interest}

The authors declare that there are no conflicts of interest regarding the publication of this paper.

\section{Acknowledgments}

This work was supported in part by the Key Project of the National Natural Science Foundation of China (no. 61134009), the National Natural Science Foundation of China (nos. 61473077, 61473078, 61503075, 61603090, and 61603089), Program for Changjiang Scholars from the Ministry of Education, and International Collaborative Project of the Shanghai Committee of Science and Technology (no. 16510711100).

\section{References}

[1] B. D. O. Anderson and J. B. Moore, Optimal Filtering, Prentice Hall, Englewood Cliffs, NJ, 1979.

[2] G. C. Goodwin, M. M. Seron, and J. A. De Doná, "Constrained Control and Estimation: An Optimisation Approach," Springer Science and Business Media, 2006.

[3] R. Dehghannasiri, M. S. Esfahani, and E. R. Dougherty, "Intrinsically bayesian robust kalman filter: an innovation process approach," IEEE Transactions on Signal Processing, vol. 65, no. 10, pp. 2531-2546, 2017.

[4] M. Ahmeid, M. Armstrong, S. Gadoue, M. Algreer, and P. Missailidis, "Real-Time Parameter Estimation of DC-DC Converters using a Self-Tuned Kalman Filter," IEEE Transactions on Power Electronics, vol. PP, no. 99, 2016.

[5] N. Davari and A. Gholami, "An asynchronous adaptive direct kalman filter algorithm to improve underwater navigation system performance," IEEE Sensors Journal, vol. 17, no. 4, pp. 1061-1068, 2017.

[6] C. V. Rao, J. B. Rawlings, and J. H. Lee, "Constrained linear state estimation - A moving horizon approach," Automatica, vol. 37, no. 10, pp. 1619-1628, 2001.

[7] C. V. Rao, J. B. Rawlings, and D. . Mayne, "Constrained state estimation for nonlinear discrete-time systems: stability and moving horizon approximations," Institute of Electrical and Electronics Engineers. Transactions on Automatic Control, vol. 48, no. 2, pp. 246-258, 2003.

[8] B.-S. Chen and W. Zhang, "Stochastic $\mathrm{H}_{2} / \mathrm{H}_{\infty}$ control with statedependent noise," IEEE Transactions on Automatic Control, vol. 49, no. 1, pp. 45-57, 2014.

[9] J. C. Willems, "Least Squares Stationary Optimal Control and the Algebraic Riccati Equation," IEEE Transactions on Automatic Control, vol. 16, no. 6, pp. 621-634, 1971.

[10] P. E. Caines and D. Q. Mayne, "On the discrete time matrix Riccati equation of optimal control," International Journal of Control, vol. 12, pp. 785-794, 1970.

[11] S. W. Chan, G. C. Goodwin, and K. . Sin, "Convergence properties of the Riccati difference equation in optimal filtering of nonstabilizable systems," Institute of Electrical and Electronics 
Engineers. Transactions on Automatic Control, vol. 29, no. 2, pp. 110-118, 1984.

[12] R. R. Bitmead, A. C. Tsoi, and P. J. Parker, "A kalman filtering approach to short-time fourier analysis," IEEE Transactions on Acoustics, Speech, and Signal Processing, vol. 34, no. 6, pp. 14931501, 1987.

[13] R. R. Bitmead, M. R. Gevers, I. R. Petersen, and R. J. Kaye, "Monotonicity and stabilizability-properties of solutions of the riccati difference equation: propositions, lemmas, theorems, fallacious conjectures and counterexamples," Systems and Control Letters, vol. 5, no. 5, pp. 309-315, 1985.

[14] C. E. de Souza, "On stabilizing properties of solutions of the Riccati difference equation," Institute of Electrical and Electronics Engineers. Transactions on Automatic Control, vol. 34, no. 12, pp. 1313-1316, 1989.

[15] R. R. Bitmead and M. Gevers, "Riccati difference and differential equations: convergence, monotonicity and stability," in The Riccati equation, Comm. Control Engrg. Ser., pp. 263-291, Springer, Berlin, 1991.

[16] D. Li, S. Kar, J. M. Moura, H. . Poor, and S. Cui, "Distributed Kalman filtering over massive data sets: analysis through large deviations of random Riccati equations," Institute of Electrical and Electronics Engineers. Transactions on Information Theory, vol. 61, no. 3, pp. 1351-1372, 2015.

[17] X. Cai, Y. Tan, S. Y. Li, and I. Mareels, "On the finite time performance of model predictive control," Systems and Control Letters, vol. 70, pp. 60-68, 2014. 


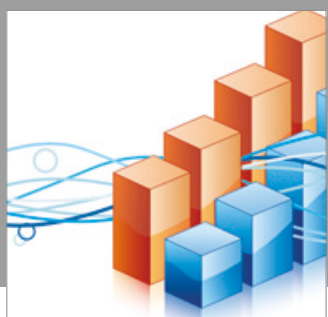

Advances in

Operations Research

vatersals

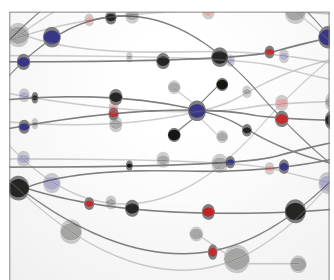

\section{The Scientific} World Journal
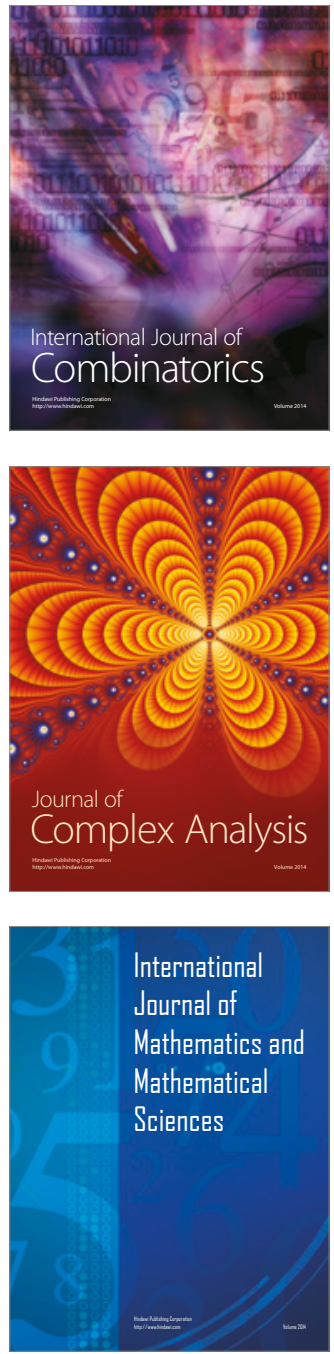
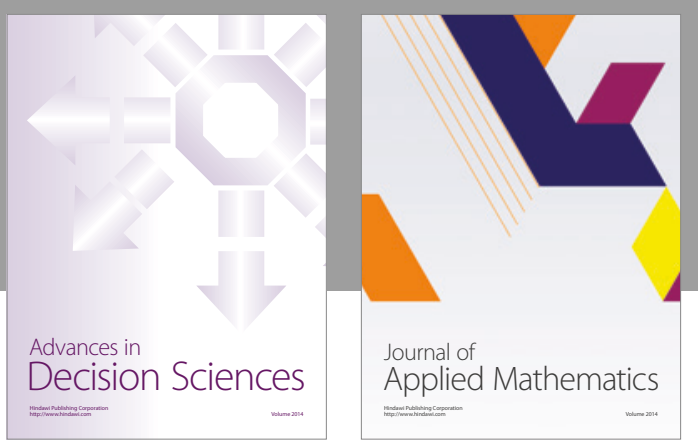

Algebra

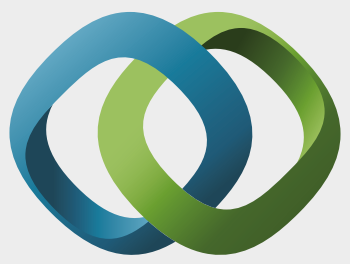

\section{Hindawi}

Submit your manuscripts at

https://www.hindawi.com
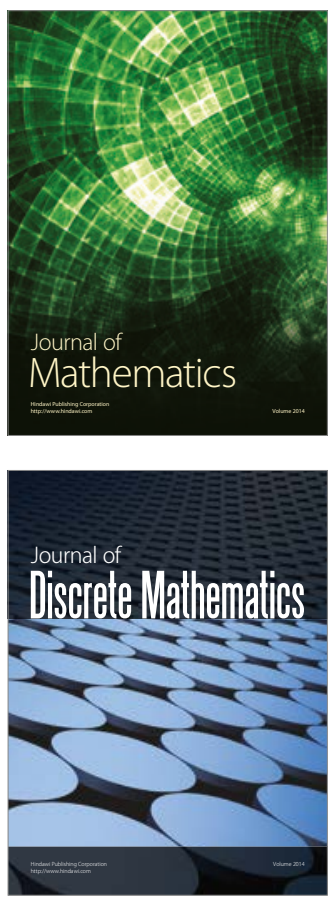

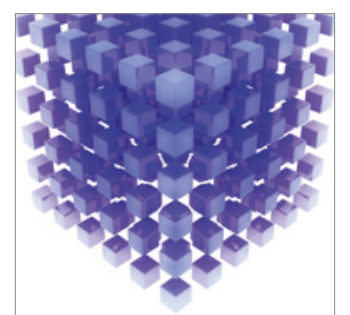

Mathematical Problems in Engineering
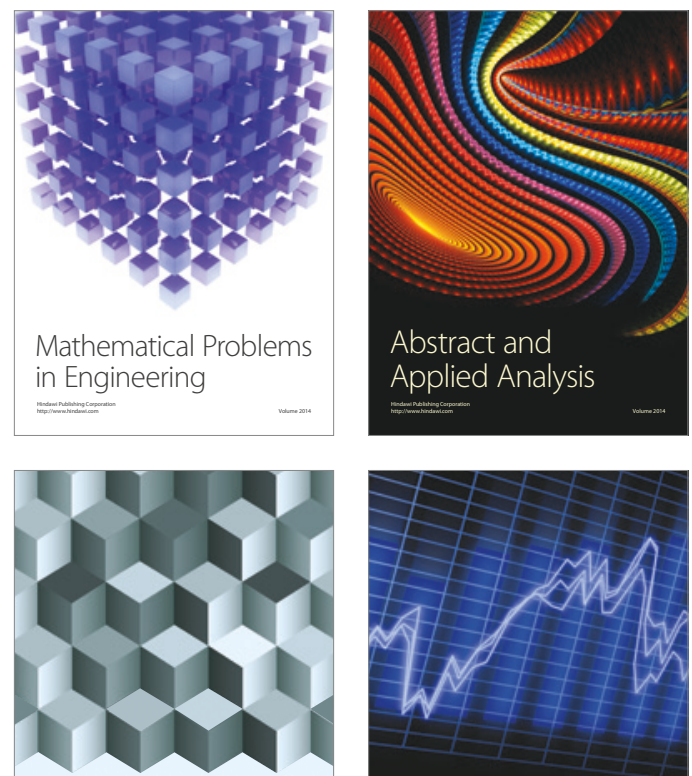

Journal of

Function Spaces

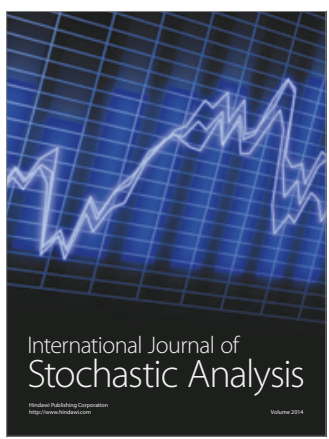

Probability and Statistics
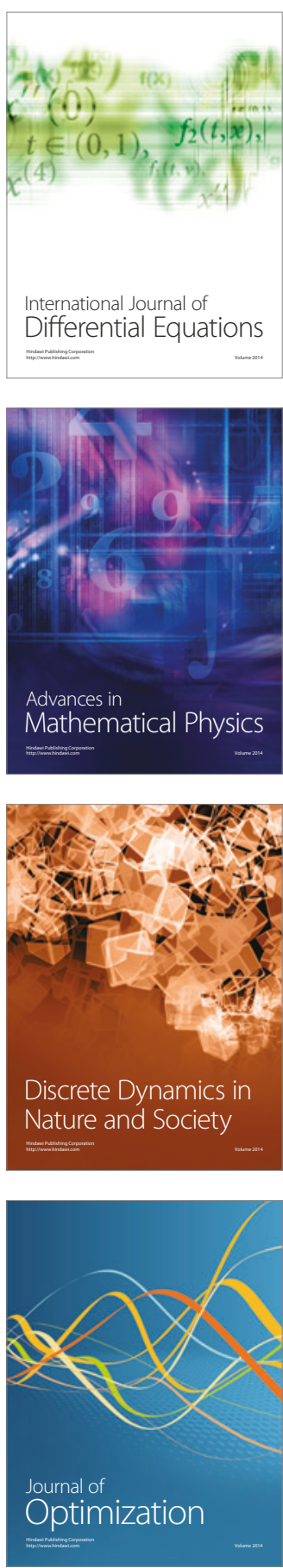